\title{
Reorientational motion and hydrogen-bond stretching dynamics in liquid water
}

\author{
H.J. Bakker *, S. Woutersen, H.-K. Nienhuys \\ FOM-Institute AMOLF, Kruislaan 407, 1098 SJ Amsterdam, Netherlands \\ Received 30 August 1999; in final form 20 April 2000
}

\begin{abstract}
The reorientational motion of the molecules in liquid water is investigated by measuring the anisotropy decay of the $\mathrm{O}-\mathrm{H}$ stretching mode of HDO dissolved in $\mathrm{D}_{2} \mathrm{O}$ using femtosecond mid-infrared pump-probe spectroscopy. We observe that the anisotropy shows a non-exponential decay with an initial fast component of which the amplitude increases with increasing lengths of the $\mathrm{O}-\mathrm{H} \cdots \mathrm{O}$ hydrogen bond. The experimental results can be accurately described with a model in which the dependence of the reorientation rate on the hydrogen-bond length and the stochastic modulation of this length are accounted for. It is found that the $\mathrm{O}-\mathrm{H}$ group of a water molecule can only reorient after the $\mathrm{O}-\mathrm{H} \cdots \mathrm{O}$ hydrogen bond has sufficiently lengthened. As a result, the effective rate of reorientation of the molecules in liquid water is determined by the rate at which the length of the hydrogen bonds is modulated. (c) 2000 Elsevier Science B.V. All rights reserved.
\end{abstract}

PACS: 77.22.-d; 78.20.-e; 03.65.-w

\section{Introduction}

Liquid water plays an important role in many chemical and biochemical processes, but little is known about the microscopic mechanism by which water affects these processes. Unfortunately, infrared absorption and Raman spectroscopy give little information on the microscopic dynamics and interactions in liquid water because the absorption and Raman bands of the low-frequency liquid modes [1-4] are strongly inhomogeneously broadened due to the strong variation in hydrogen-bond interactions $[5,6]$.

\footnotetext{
${ }^{*}$ Corresponding author. Fax: +31-20-6684106.

E-mail address: bakker@amolf.nl (H.J. Bakker).
}

In previous infrared absorption and Raman studies on water, broad Gaussian-shaped resonances near $50 \mathrm{~cm}^{-1}(1.5 \mathrm{THz}), 200 \mathrm{~cm}^{-1}(6 \mathrm{THz})$ and $650 \mathrm{~cm}^{-1}(19.5 \mathrm{THz})$ [1-4] were observed that were tentatively assigned to hydrogen-bond bend vibration, hydrogen-bond stretch vibration and librational motion, respectively $[7,8]$. These bands do not form easily distinguishable spectral species but are superimposed on a broad background that is often described as a combination of several Debye relaxational modes. The reported time constants and strengths of these relaxational modes show a large variation over the different studies. In several studies, a relatively slow relaxational mode with a time constant of approximately 8 ps has been observed [2,9-13]. In addition to this mode, one or two faster relaxational modes 
have been reported with time constants that vary between a few hundred femtoseconds to a few picoseconds [10-16]. The origin of the relaxational modes has not been unambiguously identified, and they have been assigned to the coupling between the high-frequency librational mode and lowerfrequency modes [6], to the dephasing of the stretch and bend modes of the hydrogen bonds [14] and the rotational diffusion (reorientation) of the dipolar water molecules $[15,16]$.

Recently, it has become possible to investigate the reorientational motion of liquid water in a time-resolved manner with femtosecond mid-infrared saturation spectroscopy [17]. This technique has two important advantages over conventional techniques. First, this technique directly probes the reorientation of individual molecules so that the measured (relaxational) response can only be due to the reorientation of the water molecules and cannot result from other effects like the dephasing of hydrogen-bond stretch and bend modes. Second, this technique enables the study of the orientational dynamics of selected subensembles of the water molecules, whereas in the conventional spectroscopic techniques, an average over the whole inhomogeneous ensemble is measured always.

In a recent femtosecond mid-infrared experiment, the reorientation of the individual $\mathrm{O}-\mathrm{H}$ groups of the water molecule was investigated [17]. Two distinct time scales for the reorientation of the $\mathrm{O}-\mathrm{H}$ groups were observed, depending on the strength of the $\mathrm{O}-\mathrm{H}$. . O hydrogen bond by which the water molecule is bonded to another water molecule. For $\mathrm{O}-\mathrm{H}$ groups with a strong $\mathrm{O}-\mathrm{H} \cdots \mathrm{O}$ hydrogen bond, the reorientation is slow, while for $\mathrm{O}-\mathrm{H}$ groups with a weak $\mathrm{O}-\mathrm{H} \cdots \mathrm{O}$ hydrogen bond, the reorientation is fast. These results suggest that there exist different water molecules in the liquid with different persistent strengths of the $\mathrm{O}-\mathrm{H}$... O hydrogen bond [17]. However, in other femtosecond mid-infrared pump-probe studies it was observed that the hydrogen-bonded structures cannot be persistent and that the $\mathrm{O}-\mathrm{H}$...O hydrogen bond is in fact subject to a rapid stochastic modulation [18-20]. This stochastic modulation is expected to have a strong effect on the reorientational dynamics because it induces an exchange between strong and weak $\mathrm{O}-\mathrm{H} \cdots \mathrm{O}$ hydrogen bonds. In this paper, we report a femtosecond mid-infrared study on the reorientational dynamics of liquid water in which the coupling between the hydrogen-bond stretching dynamics and the reorientational motion is investigated in detail.

\section{Experimental}

We performed both one-color and two-color pump-probe experiments on the $\mathrm{O}-\mathrm{H}$ stretch mode of a dilute solution of HDO dissolved in $\mathrm{D}_{2} \mathrm{O}(0.4 \%)$. All experiments are performed at room temperature. Mid-infrared pulses are generated via parametric generation and amplification in BBO and KTP crystals. These non-linear frequency conversion processes are pumped with the pulses delivered by a Ti:sapphire regenerative amplifier that have a central wavelength of 800 $\mathrm{nm}$, an energy per pulse of $1 \mathrm{~mJ}$ and a pulse duration of approximately $120 \mathrm{fs}$. The repetition rate of the system is $1 \mathrm{kHz}$. The generated mid-infrared pulses tunable between 2.7 and $3.3 \mu \mathrm{m}$ have a typical pulse energy of $20 \mu \mathrm{J}$ and a pulse duration of approximately $200 \mathrm{fs}$.

In the one-color experiments, a small fraction of the generated mid-infrared pulses is split off by a beam sampler and sent into a variable delay. This fraction serves as probe while the remaining part of the pulse serves as pump. In the two-color experiments, an independently tunable probe pulse is generated using a second KTP crystal. The pump and probe are focused to a common focus into the sample using a $\mathrm{CaF}_{2}$ lens. For each laser shot, the input probe energy $\left(I_{\text {ref }}\right)$ is determined. The energy of the transmitted probe light $(I)$ is also measured using a PbSe photoconductive cell. The pump beam is chopped in order to compare the transmission of the probe with $\left(T=I / I_{\text {ref }}\right)$ and without pump pulse $\left(T_{0}=I_{0} / I_{\text {ref }}\right)$. The intensity of the pump pulse is sufficient to excite a significant fraction of the HDO molecules in focus to the first excited state of the $\mathrm{O}-\mathrm{H}$ stretch vibration $\left(v_{\mathrm{OH}}=1\right)$. If the probe frequency is resonant with the $v_{\mathrm{OH}}=0 \rightarrow 1$ transition, the excitation by the pump will result in a bleaching effect for the probe $\left(\ln \left(T / T_{0}\right)>1\right)$. If the probe is resonant with the 
$v_{\mathrm{OH}}=1 \rightarrow 2$ transition, which is redshifted with respect to the $v_{\mathrm{OH}}=0 \rightarrow 1$ transition by approximately $270 \mathrm{~cm}^{-1}$ [5], the excitation by the pump will result in an induced absorption $\left(\ln \left(T / T_{0}\right)\right.$ $<1)$.

In order to study the orientational relaxation of the excited molecules, the polarization of the probe is rotated $45^{\circ}$ with respect to that of the pump using a zero-order $\lambda / 2$ plate. The transmission changes of the probe parallel to the pump $\ln (T /$ $\left.T_{0}\right)_{\|}$and perpendicular to the pump $\ln \left(T / T_{0}\right)_{\perp}$ are measured as a function of the delay $\tau$ with respect to the pump. At each laser shot, the two polarization components of the probe are simultaneously detected by splitting the transmitted probe light into two parts and by detecting these parts with separate polarizers and $\mathrm{PbSe}$ detectors.

The probability of an HDO molecule to be excited by the pump is proportional to $\cos ^{2} \theta$, with $\theta$ being the angle between the pump polarization and the molecular transition-dipole moment that is directed along the $\mathrm{O}-\mathrm{H}$ bond. The HDO molecules are randomly oriented which results in a distribution of excited molecules that is given by $f(\theta)=$ $(3 / 4 \pi) \cos ^{2} \theta$. It can easily be shown that for this distribution, the transmission change for the probe component parallel to the pump $\left(\ln \left(T / T_{0}\right)_{\|}\right)$is three times as large as the transmission change of the probe perpendicular to the pump $\left(\ln \left(T / T_{0}\right)_{\perp}\right)$. Due to the orientational relaxation, the initial angular distribution of the excited molecules $f(\theta)=(3 / 4 \pi) \cos ^{2} \theta$ decays and eventually becomes isotropic. In this limit, the signals $\ln \left(T / T_{0}\right)_{\|}$ and $\ln \left(T / T_{0}\right)_{\perp}$ are equal. Unfortunately, these signals are not only affected by the orientational dynamics but also by the decay of the excitation. The excited $v_{\mathrm{OH}}=1$ population will decay with a typical time constant of approximately $750 \mathrm{fs}$ [18]. In order to separate the orientational dynamics from the population dynamics, the dynamics of the so-called anisotropy parameter $R(\tau)$ is measured. This parameter is defined as

$$
R(\tau)=\frac{\ln \left(T(\tau) / T_{0}\right)_{\|}-\ln \left(T(\tau) / T_{0}\right)_{\perp}}{\ln \left(T(\tau) / T_{0}\right)_{\|}+2 \ln \left(T(\tau) / T_{0}\right)_{\perp}} .
$$

The vibrational relaxation affects the numerator and the denominator of this expression in exactly the same manner. As a result, $R$ is not sensitive to the vibrational relaxation and only reflects the reorientational motion. However, in the case of fast vibrational dynamics, both the numerator and the denominator decay rapidly so that a very good signal-to-noise ratio for $\ln \left(T / T_{0}\right)_{\|}$and $\ln \left(T / T_{0}\right)_{\perp}$ is required to obtain a reliable value for $R$ at large delay values.

\section{Experimental results}

In Fig. 1, the transmission changes $\ln \left(T / T_{0}\right)_{\|}$ and $\ln \left(T / T_{0}\right)_{\perp}$ are presented as a function of delay for a one-color pump-probe experiment carried out at room temperature with a central frequency of $3500 \mathrm{~cm}^{-1}$. This frequency is at the blue side of the absorption band of the $\mathrm{O}-\mathrm{H}$ stretching mode of HDO: $\mathrm{D}_{2} \mathrm{O}$. It is seen that when pump and probe overlap in time, the transmission of the probe is bleached due to the excitation of a significant part of the HDO molecules to $v_{\mathrm{OH}}=1$. The initial ratio of $\ln \left(T / T_{0}\right)_{\|}$and $\ln \left(T / T_{0}\right)_{\perp}$ is approximately 3 as expected for a randomly oriented system. For later times, both the transmission changes $\ln \left(T / T_{0}\right)_{\|}$and $\ln \left(T / T_{0}\right)_{\perp}$ decay as a result of the vibrational relaxation.

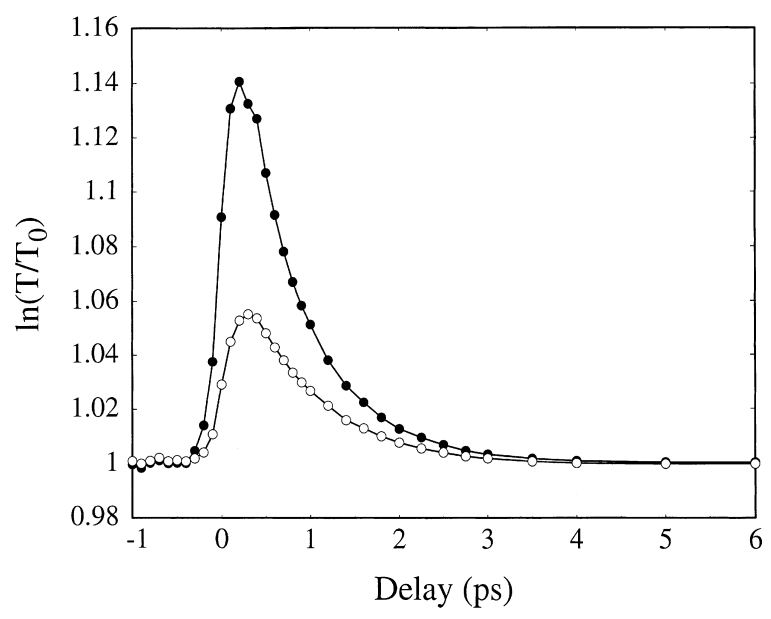

Fig. 1. Transmission changes $\ln \left(T / T_{0}\right)_{\|}$and $\ln \left(T / T_{0}\right)_{\perp}$ as a function of delay between pump and probe for a central frequency of pump and probe of $3500 \mathrm{~cm}^{-1}$. 
The resonance frequency of the $\mathrm{O}-\mathrm{H}$ stretch mode is strongly determined by the $\mathrm{O}-\mathrm{H} \cdots \mathrm{O}$ hydrogen bond. Due to the variation in length and strength of this bond, the absorption band of the $\mathrm{O}-\mathrm{H}$ stretch mode is strongly inhomogeneously broadened. With decreasing hydrogen-bond lengths (and increasing hydrogen-bond strengths), the absorption frequency of the $\mathrm{O}-\mathrm{H}$ stretch vibration decreases. In Fig. 2, the delay dependence of the normalized anisotropy parameter $R(\tau)$ is presented for three one-color pump-probe experiments with central frequencies of 3320,3400 and $3500 \mathrm{~cm}^{-1}$. These frequencies correspond with the red, central, and blue parts of the absorption band of the $\mathrm{O}-\mathrm{H}$ stretching mode of $\mathrm{HDO}: \mathrm{D}_{2} \mathrm{O}$. The laser spectra and the absorption spectrum of the $\mathrm{O}-\mathrm{H}$ stretching mode of HDO: $\mathrm{D}_{2} \mathrm{O}$ are presented in Fig. 3. In Fig. 4, the decay of the anisotropy is presented for a two-color pump-probe experiment in which the probe is resonant with the $v_{\mathrm{OH}}=$ $1 \rightarrow 2$ transition.

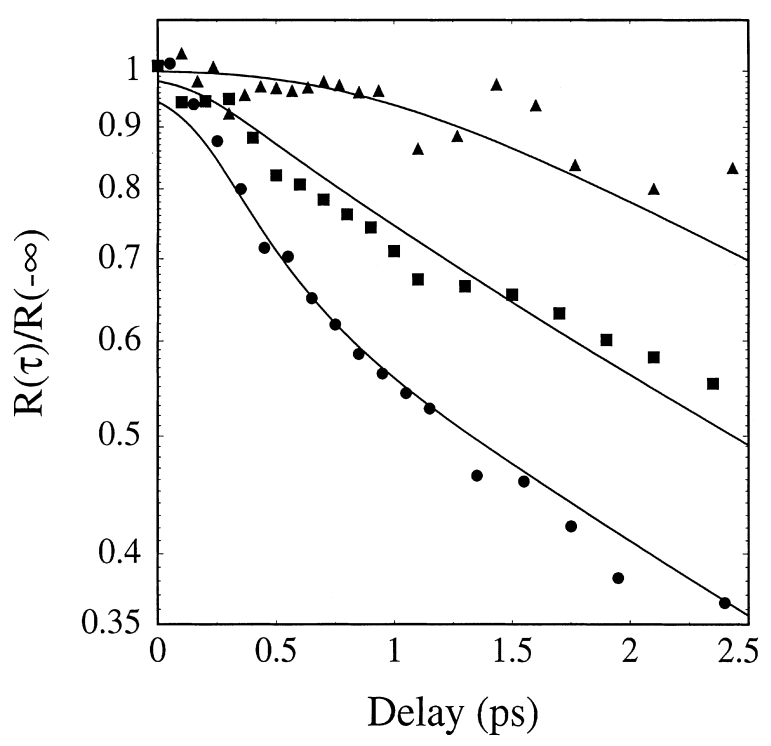

Fig. 2. Normalized anisotropy parameter $R$ as a function of delay between pump and probe for three different central pump and probe frequencies: $3320 \mathrm{~cm}^{-1}(\mathbf{\Lambda}), 3400 \mathrm{~cm}^{-1}(\boldsymbol{\square})$ and 3500 $\mathrm{cm}^{-1}(\mathbf{O})$. The data at $3500 \mathrm{~cm}^{-1}$ correspond to the delay scans of Fig. 1. The solid lines represent the results of calculations using the model described in Section 4.

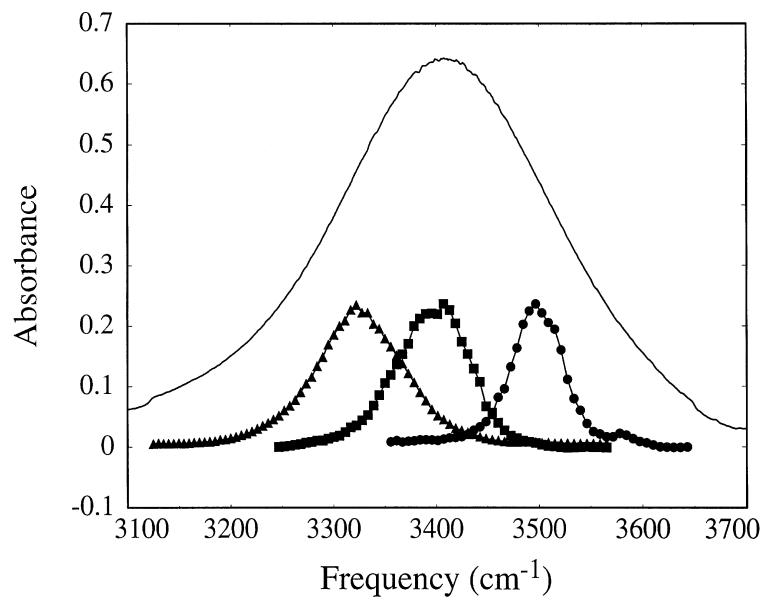

Fig. 3. Pulse spectra of the laser pulses used to obtain the data shown in Fig. 2. Also shown is the absorption spectrum of the $\mathrm{O}-\mathrm{H}$ stretching mode of $\mathrm{HDO}$ dissolved in $\mathrm{D}_{2} \mathrm{O}(0.4 \%)$.

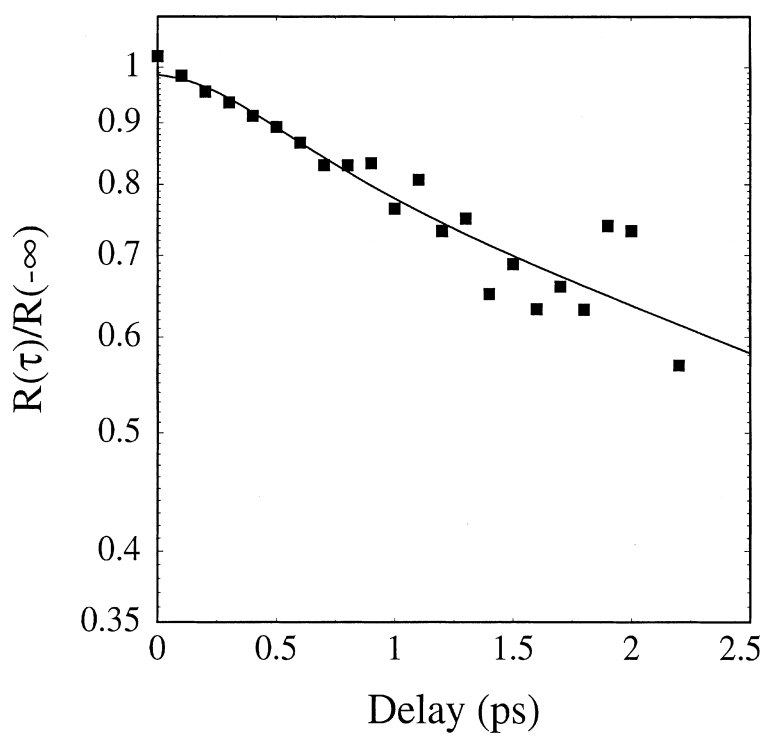

Fig. 4. Normalized anisotropy parameter $R$ as a function of delay between pump and probe for a pump frequency of 3400 $\mathrm{cm}^{-1}$ and a probe frequency of $3100 \mathrm{~cm}^{-1}$. The probe pulse is resonant with the $v_{\mathrm{OH}}=1 \rightarrow 2$ transition. The solid lines represent the results of calculations using the model described in Section 4 .

\section{Model}

In the following, we present a model for liquid water by which the observations of Section 3 can 
be explained. In this model, both the frequency dependence of the reorientation and the stochastic stretching dynamics of the $\mathrm{O}-\mathrm{H} \cdots \mathrm{O}$ hydrogen bond are accounted for.

\subsection{Frequency-dependent reorientation}

For an isolated molecule as light as HDO, the classical angular frequency of the rotation is on the order of $10 \mathrm{ps}^{-1}$ at room temperature. The much slower observed decay of the anisotropy in Fig. 2 shows that in the liquid phase, the reorientation must be strongly hindered. This is to be expected since a water molecule has to break its hydrogen bonds in order to change its orientation. Recent measurements of the reorientation rate at different temperatures showed that the reorientation rate strongly increases with temperature. If the reorientation is described as an activated process the following Arrhenius equation for the reorientation time results:

$\frac{1}{\tau_{\text {rot }}(v)}=\frac{1}{\tau_{0}} \mathrm{e}^{-E_{\text {act }}(v) / k_{\mathrm{B}} T}$

with $\tau_{\text {rot }}(v)$ being the reorientation time constant; $v$, the $\mathrm{O}-\mathrm{H}$ stretch frequency; $\tau_{0}$, a prefactor; $E_{\text {act }}(v)$, the activation energy; $k_{\mathrm{B}}$, the Boltzmann's constant and $T$, the temperature. The data presented in Fig. 2 show that the reorientation rate strongly depends on the resonance frequency of the $\mathrm{O}-\mathrm{H}$ stretching mode. This can be expected since a lower $\mathrm{O}-\mathrm{H}$ stretch frequency implies a stronger $\mathrm{O}-\mathrm{H} \cdots \mathrm{O}$ hydrogen bond and thus a slower reorientation. Hence, the activation energy will depend on the hydrogen-bond strength and is expected to decrease with increasing $\mathrm{O}-\mathrm{H}$ stretch frequencies.

\subsection{Stochastic stretching dynamics of the hydrogen bonds}

In several studies, it has been found that the effects of hydrogen bonding on the absorption band of a high-frequency vibration can be well described with an adiabatic model in which the potential-energy function of the low-frequency hydrogen-bond mode is determined by the quantum state of a high-frequency mode (in this case, the $\mathrm{O}-\mathrm{H}$ stretching mode) [20-27]. In this description, the potential-energy function of the hydrogen-bond mode is often assumed to be a harmonic potential that has exactly the same shape for the ground and excited states of the high-frequency $\mathrm{O}-\mathrm{H}$ stretching mode [20-25]. These potentials are displaced with respect to each other which results (in the classical limit) in a Gaussianshaped absorption spectrum with a width that increases with increasing displacements.

In the liquid phase, the hydrogen-bond mode will strongly interact with other degrees of freedom. These interactions can be accounted for by describing the hydrogen-bond coordinate as being subject to a stochastic modulation process. This stochastic process is often described as a GaussMarkov process [20,25,26,28]. The overall model of displaced parabolic functions and Gauss-Markov stochastic modulation is often denoted as the Brownian oscillator model [28]. In two recent experimental studies, it was found that the Brownian oscillator model provides an accurate description of both the linear absorption spectrum and the spectral diffusion of the $\mathrm{O}-\mathrm{H}$ stretch vibration of $\mathrm{HDO}$ dissolved in $\mathrm{D}_{2} \mathrm{O}[19,20]$.

In the present calculation, we will employ the Brownian oscillator model to describe the absorption spectrum and the stochastic modulation of the hydrogen-bond mode. In this respect, our approach is exactly the same as has been used before in the modeling of transient spectra of hydrogen-bonded $\mathrm{O}-\mathrm{H}$ stretch vibrations. The main extension is that we will also incorporate the dependence of the reorientation rate on the hydrogen-bond length, in order to model the decay of the anisotropy.

In Fig 5, the harmonic potentials of the hydrogen-bond mode are presented that are used in the calculation. The curvature of these potentials is chosen such that for the resonance frequency of the hydrogen-bond stretching mode a value of 200 $\mathrm{cm}^{-1}$ results. This gives the following dependence for the hydrogen-bond potential energy $V_{i}$ in $\mathrm{cm}^{-1}$ of the state $v_{\mathrm{OH}}=i$ on the hydrogen-bond coordinate $r$ in $\mathrm{m}: V_{i}=(a / 100 h c)\left(r-r_{i}\right)^{2}$ with $a=$ $10.68 \mathrm{~kg} \mathrm{~s}^{-2}, h$ is the Planck's constant, $c$, the 


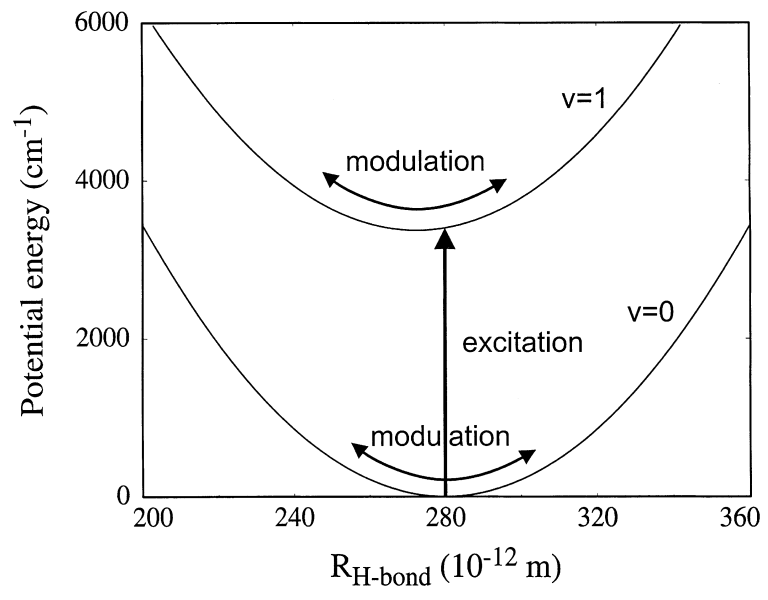

Fig. 5. Potential-energy curves of the hydrogen-bond mode for the $v_{\mathrm{OH}}=0$ and $v_{\mathrm{OH}}=1$ states. These curves are used in the calculation of the stochastic modulation of the hydrogen-bond length. The curvature of the potential energy curves is chosen such that a resonance frequency for the hydrogen-bond stretching mode of $200 \mathrm{~cm}^{-1}$ results. The potential energy curve of the $v_{\mathrm{OH}}=1$ state is displaced by $-7.35 \times 10^{-12} \mathrm{~m}$ with respect to the $v_{\mathrm{OH}}=0$ potential. This leads to a full width at half maximum of the linear absorption spectrum of $260 \mathrm{~cm}^{-1}$ and a dynamic Stokes shift of $60 \mathrm{~cm}^{-1}$.

velocity of light in vacuum and $r_{i}$, the minimum of the potential $V_{i}$. For $r_{0}$, we use a value of $280 \times 10^{-12} \mathrm{~m}$ [29]. The relative displacement $r_{0}-r_{1}=7.35 \times 10^{-12} \mathrm{~m}$. This displacement leads to an absorption spectrum with a full width at half maximum (FWHM) of $260 \mathrm{~cm}^{-1}$, in agreement with the experimental spectrum shown in Fig. 3.

The interactions with the surrounding molecules lead to rapid dynamics of the hydrogen bond in the potential of $v_{\mathrm{OH}}=0$. These dynamics in turn induce a rapid spectral diffusion of the $\mathrm{O}-\mathrm{H}$ stretching frequency of the dissolved HDO molecules. Hence, the frequency of each $\mathrm{O}-\mathrm{H}$ oscillator is given by

$\omega_{01}(t)=\omega_{0}+\delta \omega_{01}(t)$

with $\omega_{01}(t)$ being the frequency of the $v_{\mathrm{OH}}=0 \rightarrow 1$ transition at time $t, \omega_{0}$, the central frequency of this transition and $\delta \omega_{01}(t)$, the detuning at time $t$. Because the absorption spectrum of the $\mathrm{O}-\mathrm{H}$ stretching mode is nearly Gaussian, the motion of the hydrogen bond can assumed to be overdamped [28]. As a result, the spectral dynamics of the $\delta \omega_{01}(t)$ follows a Gauss-Markov random process, with an autocorrelation function

$\left\langle\delta \omega_{01}(t) \delta \omega_{01}(0)\right\rangle=\Delta^{2} \mathrm{e}^{-t / \tau_{c}}$

with $\tau_{\mathrm{c}}$ being the autocorrelation time constant for the detuning. Recently, values for $\tau_{\mathrm{c}}$ of 700 [19] and $500 \mathrm{fs}$ [20] have been reported. These values are in quite good agreement with the time constant of $500 \mathrm{fs}$ for breaking the hydrogen bond that has been inferred from depolarized Rayleigh scattering experiments [15] and also agree quite well with the results of recent molecular dynamics simulations [30]. In modeling the data of Figs. 2 and 4, we will use a value for $\tau_{c}$ of 500 fs to characterize the hydrogen-bond dynamics in both the $v_{\mathrm{OH}}=0$ state and the $v_{\mathrm{OH}}=1$ state.

Due to the relative displacement of the potentials, the excitation from the minimum of the hydrogen-bond potential corresponding to the $v_{\mathrm{OH}}=0$ state leads to a non-equilibrium hydrogenbond length in the hydrogen-bond potential corresponding to the $v_{\mathrm{OH}}=1$ state. Equilibration in the potential of the latter state results in contraction of the hydrogen bond and to a redshift in the stimulated emission from $v_{\mathrm{OH}}=1$ to $v_{\mathrm{OH}}=0$. This redshift (dynamic Stokes shift) has indeed been observed and has a value of approximately 60 $\mathrm{cm}^{-1}$ [20]. It should be noted that, in the case of harmonic potentials, a direct relationship exists between the Stokes shift $S$ and the parameter $\Delta$ that determines the width of the absorption line shape $\mathrm{e}^{-\omega^{2} / 2 \Delta^{2}}$ [28]:

$S=\hbar \Delta^{2} / k_{\mathrm{B}} T$

with $\hbar$ being the Dirac's constant (Planck's constant divided by $2 \pi$ ).

In Fig. 6, the calculated Gaussian spectral shapes for the absorption $v_{\mathrm{OH}}=0 \rightarrow 1$ and the stimulated emission $v_{\mathrm{OH}}=1 \rightarrow 0$ are presented. The latter spectrum is redshifted by $60 \mathrm{~cm}^{-1}$ with respect to the first due to the Stokes shift. The width and shape of the absorption spectrum correspond quite well to the experimental spectrum, as shown in Fig. 3. 


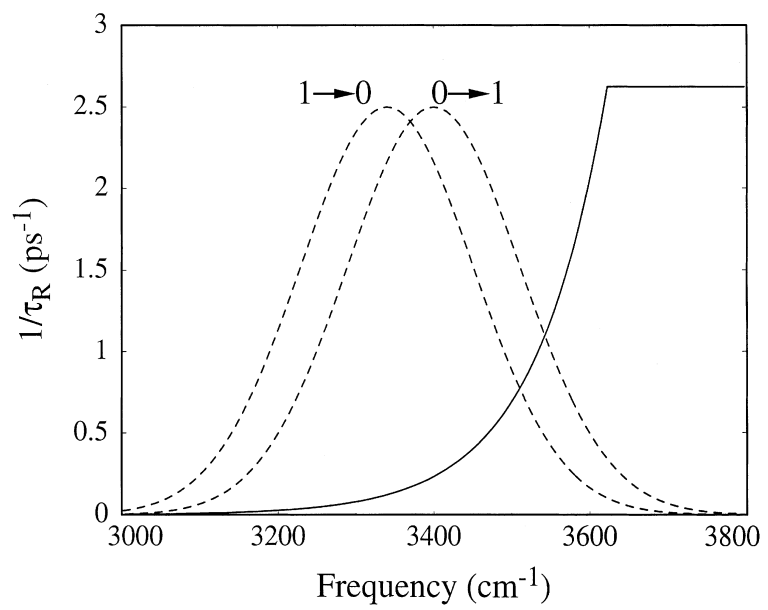

Fig. 6. Reorientation rate $1 / \tau_{\mathrm{R}}$ as a function of the frequency of the $\mathrm{O}-\mathrm{H}$ stretching mode (-). The reorientation rate strongly increases with frequency and increasing hydrogen-bond length. Also shown are the calculated absorption band $\left(v_{\mathrm{OH}}=0 \rightarrow 1\right)$ and the stimulated emission band $\left(v_{\mathrm{OH}}=1 \rightarrow 0\right)$ of the $\mathrm{O}-\mathrm{H}$ stretching mode (- - -).

\subsection{Method of calculation}

The Brownian oscillator model can be used to derive explicit analytic solutions for the isotropic transient spectrum of the $\mathrm{O}-\mathrm{H}$ stretching mode at different delay times $[24,28]$. In order to calculate the anisotropy decay, this model should be extended with an explicit description of the dependence of the reorientation rate on the hydrogenbond length, as is presented in Section 4.1. This combination of the Brownian oscillator model and the dependence of the reorientation rate on the hydrogen-bond length is too complicated for an analytical evaluation so that a numerical approach is required. In this numerical approach, the hydrogen-bond coordinate $r$ of the $v_{\mathrm{OH}}=0$ and the $v_{\mathrm{OH}}=1$ states is divided into bins that each correspond to a particular length interval, namely $1 \times 10^{-12} \mathrm{~m}$, of the hydrogen bond. These bins exchange population to account for the stochastic stretching dynamics of the hydrogen-bond length. The exchange of population is calculated in the following way: In each time step of the integration, the bins exchange population with their nextnearest neighbors with a time constant that is given by $\tau_{s}= \begin{cases}\tau_{h} \mathrm{e}^{100 h c \Delta V / k_{\mathrm{B}} T}, & \Delta V>0, \\ \tau_{h}, & \Delta V<0\end{cases}$

with $\Delta V$ being the difference in potential energy in $\mathrm{cm}^{-1}$ at the central positions of the bins. Eventually, this population exchange process results in a Boltzmann distribution for which the harmonic potential $V_{0}$ corresponds to a Gaussian distribution in the position coordinate $r$ around $r_{0}$. The time constant $\tau_{h}$ is related to the autocorrelation time constant $\tau_{\mathrm{c}}$ via $\tau_{h}=\tau_{\mathrm{c}}(1-\gamma)$ [31]. The parameter $\gamma$ depends on the width of the Gaussian equilibrium distribution: $\gamma^{2}=1-4 / w^{2}$, with $w$ being the half width (in bins) at $1 / e$ of the maximum of the equilibrium distribution. In our calculation, $w$ being was equal to 21 bins, and thus $\gamma=0.9954$ and $\tau_{h}=2.3 \mathrm{fs}$. This large value of $\gamma$ implies that the stochastic modulation of the hydrogen-bond length is truly modeled as a Gaussian process since the change in hydrogen-bond length $r$ resulting from a single modulation event is small compared to the width of the Gaussian distribution in $r$ [31].

To account for the anisotropy decay of the population in these bins, the frequency-dependent reorientation rate is transformed to a reorientation rate that depends on the hydrogen-bond length $r$. This transformation can easily be performed since for displaced harmonic potentials, the transition frequency $v$ is linearly related to $r$ as

$v=(2 a / 100 h c)\left(r-r_{0}\right)\left(r_{0}-r_{1}\right)+v_{0}$

with $v$ in $\mathrm{cm}^{-1}, r$ and $r_{0}$ in $\mathrm{m}, a=10.68 \mathrm{~kg} \mathrm{~s}^{-2}$, $\left(r_{0}-r_{1}\right)=7.35 \times 10^{-12} \mathrm{~m}$ and $v_{0}$, the center frequency of the absorption band, which is 3400 $\mathrm{cm}^{-1}$. The reorientation rate is discretized to define a reorientation rate for each bin (length interval) of the hydrogen bond in the $v_{\mathrm{OH}}=0$ and $v_{\mathrm{OH}}=1$ states.

The time dependence of the anisotropy is evaluated in the following way: The excitation by the pump will result in a hole $\Delta n_{0}(r)$ in the population distribution of $v_{\mathrm{OH}}=0$ and to an induced population distribution $\Delta n_{1}(r)$ in $v_{\mathrm{OH}}=1$. The hydrogen-bond position, $r$, at which the hole in the $v_{\mathrm{OH}}=0$ state and the population in the $v_{\mathrm{OH}}=1$ state are generated and is fully determined by the central frequency and the spectral width of the 
pump pulse. The signals $\ln \left(T / T_{0}\right)_{\|}$and $\ln \left(T / T_{0}\right)_{\perp}$, as measured by the parallel and perpendicular polarization components of the probe pulse, are

$\ln \left(T / T_{0}\right)_{\|} \propto \int I_{\text {probe }}(r)\left(\Delta n_{1, \|}(r)-\Delta n_{0, \|}(r)\right) \mathrm{d} r$,

$\ln \left(T / T_{0}\right)_{\perp} \propto \int I_{\text {probe }}(r)\left(\Delta n_{1, \perp}-\Delta n_{0, \perp}\right) \mathrm{d} r$

with $\Delta n_{i, \|}(r)$ and $\Delta n_{i, \perp}(r)$ being the change of the population distribution of the state $v_{\mathrm{OH}}=i$ as experienced by the parallel and perpendicular polarization components of the probe, respectively, and $I_{\text {probe }}(r)$, the normalized probe intensity as a function of the hydrogen-bond coordinate $r$. The latter function can be obtained from $I_{\text {probe }}(v)$ using Eq. (4.6).

$$
R(t)=\frac{\int I_{\text {probe }}(r)\left(\Delta n_{\text {ani }, 1}(r, t)-\Delta n_{\text {ani }, 0}(r, t)\right) \mathrm{d} r}{\int I_{\text {probe }}(r)\left(\Delta n_{\text {iso }, 1}(r, t)-\Delta n_{\text {iso }, 0}(r, t)\right) \mathrm{d} r} .
$$

The time dependence of pump and probe is accounted for by generating the initial $\Delta n_{\text {iso }, 0}(r)$, $\Delta n_{\text {ani, } 0}(r), \Delta n_{\text {iso, } 1}(r)$, and $\Delta n_{\text {ani, } 1}(r)$ with a time profile that is given by the cross-correlation of pump and probe.

\section{Results and interpretation}

The solid curves in Figs. 2 and 4 represent the delay dependence of the anisotropy calculated with the model presented in Section 4 . The best fit of the experimental results is obtained with the following dependence of the activation energy $E_{\text {act }}(v)$ in $\mathrm{cm}^{-1}$ on the distance $r$ in $\mathrm{m}$ :

$E_{\text {act }}(r)= \begin{cases}1115-2 \times 10^{13}\left(r-r_{0}\right), & \left(r-r_{0}\right) \leqslant 26 \times 10^{-12} \mathrm{~m}, \\ 600, & \left(r-r_{0}\right) \geqslant 26 \times 10^{-12} \mathrm{~m} .\end{cases}$

The evaluation of the anisotropy parameter can be simplified by defining the so-called anisotropic population distributions

$\Delta n_{\mathrm{ani}, i}(r)=\Delta n_{i, \|}(r)-\Delta n_{i, \perp}(r)$,

and isotropic population distributions

$\Delta n_{\mathrm{iso}, i}(r)=\Delta n_{i, \|}(r)+2 \Delta n_{i, \perp}(r)$

with $i$ denoting either the $v_{\mathrm{OH}}=0$ or the $v_{\mathrm{OH}}=1$ state. The isotropic population distributions $\Delta n_{\text {iso }, 0}(r)$ and $\Delta n_{\text {iso, } 1}(r)$ are only affected by the stochastic modulation of the hydrogen-bond length, whereas the anisotropic distributions $\Delta n_{\text {ani, },}(r)$ and $\Delta n_{\text {ani, } 1}(r)$ are affected by both the stochastic stretching dynamics and the reorientation. At each time point, the isotropic and anisotropic population distributions are evaluated using a fourth-order Runge-Kutta scheme.

The anisotropy parameter $R$ at a certain time is given by
This activation energy agrees quite well with the energetic cost of hydrogen-bond breaking in water for which a value of approximately $900 \mathrm{~cm}^{-1}$ has been inferred using Raman spectroscopy [32]. It also agrees quite well with the value of approximately $650 \mathrm{~cm}^{-1}$ for the activation energy of the rotation diffusion of water that was inferred from quasi-elastic incoherent neutron scattering [33].

The frequency dependence of the reorientation rate $1 / \tau_{\mathrm{R}}$ that results from Eq. (5.1) is shown in Fig. 6. The rate is low at low frequencies, strongly increases in a particular frequency regime and is at a constant high value at high frequencies. From Fig. 6, it can be seen that this frequency-independent high value is reached at a frequency of 3650 $\mathrm{cm}^{-1}$. At this frequency, the reorientation is no longer hindered by the $\mathrm{O}-\mathrm{H} \cdots \mathrm{O}$ hydrogen bond. The cut-off frequency of $3650 \mathrm{~cm}^{-1}$ is indeed close to the gas-phase value of the $\mathrm{O}-\mathrm{H}$ stretch frequency of the isolated HDO molecule. The residual activation energy of $600 \mathrm{~cm}^{-1}$ results from 
steric hindering by surrounding $\mathrm{D}_{2} \mathrm{O}$ molecules and other hydrogen bonds that negligibly affect the $\mathrm{O}-\mathrm{H}$ stretch frequency. The pre-exponential time constant $\tau_{0}$ has a value of $20 \mathrm{fs}$ which is on the order of the rotation time of the free HDO molecule. This value agrees quite well with the value of 48.5 fs for the pre-exponential time constant that was inferred from quasi-elastic incoherent neutron scattering [33].

It is clear from Figs. 2 and 4 that at least two distinct reorientational time scales can be distinguished. In fact, on the basis of the model of Section 4, even three different reorientational time constants are expected. The first, fast time constant is mainly observed at the blue side of the absorption band in the first picosecond after excitation and is associated with the immediate reorientation of excited molecules for which the $\mathrm{O}-\mathrm{H} \cdots \mathrm{O}$ hydrogen bond is long. These molecules can directly change orientation with a time constant that is equal to the high-frequency limit of the reorientation rate shown in Fig. 6. After 1 ps, most of these molecules have reoriented and the stochastic stretching dynamics of the hydrogen-bond length has led to an equilibration of the population distributions $\Delta n_{\text {iso }, 0}, \Delta n_{\text {iso }, 1}, \Delta n_{\text {ani }, 0}$, and $\Delta n_{\text {ani, } 1}$.

After equilibration, the isotropic populations $\Delta n_{\text {iso }, 0}$ and $\Delta n_{\text {iso, } 1}$ have acquired a permanent Gaussian shape centered at the minima of the potentials of the $v_{\mathrm{OH}}=0$ and $v_{\mathrm{OH}}=1$ states. In comparison with these isotropic distributions, the equilibrated anisotropic distributions $\Delta n_{\text {ani, } 0}$ and $\Delta n_{\text {ani, } 1}$ will be shifted to smaller values of $r$ because the decay rate of the anisotropy increases with $r$. From Eq. (4.11), it is clear that these equilibrated distributions result in an anisotropy parameter $R$ that is smaller at the blue side (large values of $r$ ) than at the red side. However, the time dependence of the $R$ will be the same at all probe frequencies after equilibration, because the shape of the population distributions no longer changes. Hence, after $1 \mathrm{ps,}$, the amplitude of $R$ is much smaller at the blue side than at the red side of the absorption band, but the decay rate of $R$ is the same at all probe frequencies as is indeed observed in Fig. 2.

The second and third time constants of the reorientation are associated with the decay of the equilibrated anisotropic population distributions in the $v_{\mathrm{OH}}=0$ and $v_{\mathrm{OH}}=1$ states, respectively. In both states, these distributions are concentrated at values of $r$ at which the reorientation rate by direct breaking of the hydrogen bond is negligible. Hence, the hydrogen-bond should first be elongated before reorientation can occur. The resulting reorientation will become faster with increasing fractions of fast reorienting molecules and decreasing time constants for the reorientation and the stochastic modulation of the hydrogen bond.

In the $v_{\mathrm{OH}}=1$ state, the minimum of the potential energy curve and the maximum of the equilibrated $\Delta n_{\text {ani, } 1}(r)$ population distribution are at a smaller value of $r$ than in the $v_{\mathrm{OH}}=0$ state. Since the direct reorientation rate is assumed to depend only on the hydrogen-bond length, the fraction of molecules for which the direct reorientation is fast will be smaller in the $v_{\mathrm{OH}}=1$ state than in the $v_{\mathrm{OH}}=0$ state. This can clearly be seen in Fig. 6: for the stimulated emission band $v_{\mathrm{OH}}=1 \rightarrow 0$, the overlap with the spectral region in which the reorientation is fast is much smaller than for the absorption band $v_{\mathrm{OH}}=0 \rightarrow 1$. As a result, the effective reorientation time constant after equilibration will be longer for the $v_{\mathrm{OH}}=1$ state than for the $v_{\mathrm{OH}}=0$ state. The decay observed in Fig. 2 in the time interval between 1 and 2.5 ps results from both the decay of the equilibrated population in the $v_{\mathrm{OH}}=0$ state and the decay of the equilibrated population in the $v_{\mathrm{OH}}=1$ state. In the experiment of Fig. 4, the probe pulse is resonant with the $v_{\mathrm{OH}}=1 \rightarrow 2$ transition so that only the relatively long effective reorientation time constant of the equilibrated population $v_{\mathrm{OH}}=1$ state is observed. Indeed, in Fig. 4, the decay rate of $R$ is somewhat slower than in Fig. 2.

From our results, it can be concluded that the reorientational dynamics in liquid water are strongly coupled to the rapid stochastic stretching dynamics of the hydrogen bond. In our model, this coupling is largely accounted for: the model explicitly describes the enabling (disabling) of reorientation due to the lengthening (shortening) of the hydrogen bond. However, the coupling will in principle also work in the other direction: reorientation by breaking a hydrogen bond will influence the length of the hydrogen bond. This effect 
will be small because the stochastic modulation of the hydrogen-bond length is a much faster process than the reorientation. Hence, the reorientation is strongly affected by the fast stochastic modulation of the hydrogen-bond length, whereas the modulation of the hydrogen-bond length is hardly influenced by the slow reorientation.

\section{Comparison with other studies}

In previous Raman, dielectric relaxation and far-infrared spectroscopic studies of water, a relaxational response was measured, which likely results from the reorientation of the water molecules. In these studies, the complex dielectric function $\epsilon(\omega)$ is measured at low frequencies. Thereby, the decay of the first-order correlation function $C_{1}(t)=\langle\mathbf{P}(t) \cdot \mathbf{P}(0)\rangle$ is measured, with $\mathbf{P}$ being the macroscopic polarization. Often, this correlation function is assumed to be a sum of exponentially decaying functions: $\langle\mathbf{P}(t) \cdot \mathbf{P}(0)\rangle=$ $\sum_{j} \exp \left(-t / \tau_{1, j}\right)$ with $\tau_{1, j}$ being the relaxation time constants, often denoted as the Debye relaxation time constants.

In the femtosecond experiments presented here, the second-order correlation function $C_{2}(t)=$ $\left\langle P_{2}(\mathbf{e}(t) \cdot \mathbf{e}(0))\right\rangle=\mathrm{e}^{-t / \tau_{2}}$, with $P_{2}(x)$ being the second Legendre polynomial in $x$ and e a unit vector in the molecular frame. In our experiments, the dynamics of the $\mathrm{O}-\mathrm{H}$ stretch vibration of the HDO molecule is probed so that this unit vector points along the $\mathrm{O}-\mathrm{H}$ bond. If the dynamics of this unit vector is the same as that of the macroscopic polarization and if there is no preferred rotation axis or molecular orientation, i.e. when the reorientation is isotropic, it can easily be shown that the time constants $\tau_{1}$ and $\tau_{2}$ relate as $\tau_{1}=3 \tau_{2}$ [34]. It should be noted that the reorientation time of the $\mathrm{O}-\mathrm{H}$ bond can be different from that of the dipole of the HDO molecule and that the dynamics of an individual dipole can differ from that of the macroscopic polarization. In this latter macroscopic response, local-field effects can play an important role. These local-field effects tend to sharpen up the dielectric response, thereby making the ratio of $\tau_{1}$ and $\tau_{2}$ larger than 3 . However, in order to make a comparison of our results with dielectric relax- ation and far-infrared spectroscopic studies, we will assume that the relaxation of $\langle\mathbf{P}(t) \cdot \mathbf{P}(0)\rangle$ is three times as slow as the decay of the anisotropy of the $\mathrm{O}-\mathrm{H}$ stretching mode. Under this assumption, the model described in Section 4 can be used to calculate the time dependence of the correlation function $\langle\mathbf{P}(t) \cdot \mathbf{P}(0)\rangle$ as would be measured in dielectric relaxation and far-infrared spectroscopic experiments. In this calculation of $\langle\mathbf{P}(t) \cdot \mathbf{P}(0)\rangle$, we neglect the population of the $v_{\mathrm{OH}}=1$ state since in these experiments, all molecules will be in the ground state of the $\mathrm{O}-\mathrm{H}$ stretching mode. In addition, there is no initial frequency dependence induced by a pump pulse that is spectrally narrower than the absorption line width. Hence, the initial thermal population distributions $\Delta n_{\text {iso }, 0}(r)$ and $\Delta n_{\text {ani, },}(r)$ will be Gaussian.

The calculated $\langle\mathbf{P}(t) \cdot \mathbf{P}(0)\rangle$ is presented in Fig. 7. After an initial fast decay, the relaxation becomes exponential with a time constant of $7.85 \mathrm{ps}$. This exponential component forms the dominant contribution to the relaxation function. This purely exponential decay appears after a time of approximately $1 \mathrm{ps}$, corresponding to the characteristic time scale of the modulation of the hydrogen-bond length. The calculated decay of $\langle\mathbf{P}(t) \cdot \mathbf{P}(0)\rangle$ is in excellent agreement with the relaxational response that is observed in dielectric relaxation [10] and far-infrared ( $\mathrm{THz})$ spectroscopic studies [2,9,11-13]. This good agreement strongly supports an interpretation of the experimentally observed relaxational response in terms of the reorientation of the dipolar water molecules. For the slow component, an experimental Debye time constant of approximately 8 ps has been reported, which compares well with the time constant of 7.85 ps that follows from our calculation.

In Fig. 7b, the fast initial decay of $\langle\mathbf{P}(t) \cdot \mathbf{P}(0)\rangle$ is highlighted by taking the difference between $\langle\mathbf{P}(t) \cdot \mathbf{P}(0)\rangle$ and an exponential decay with a time constant of $7.85 \mathrm{ps}$. This fast initial decay results from water molecules for which the $\mathrm{O}-\mathrm{H} \cdots \mathrm{O}$ is long at the moment the (static) electric field is switched. After approximately $1 \mathrm{ps,} \mathrm{these} \mathrm{mole-}$ cules have reoriented, and the much slower decay of the remaining molecules is observed. The fast initial decay can be fitted to an exponential function with a time constant of 600 fs. For this 

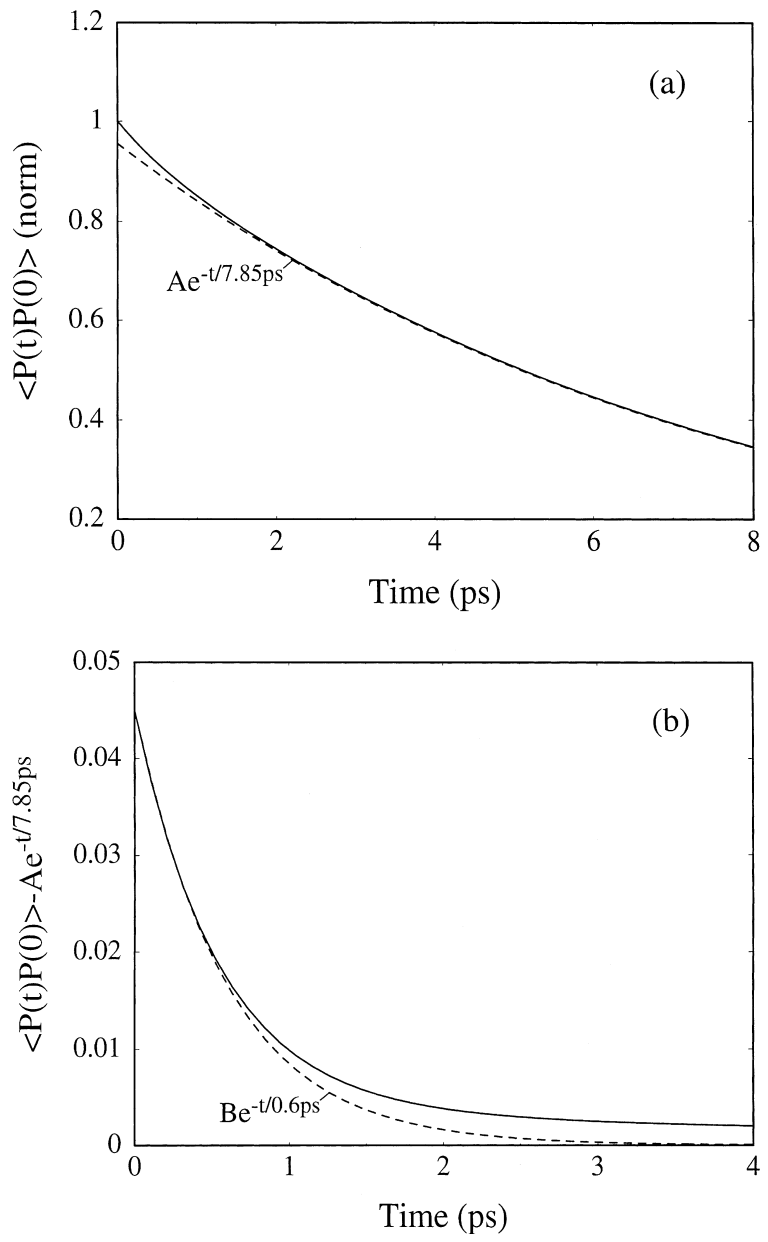

Fig. 7. Calculated $\langle\mathbf{P}(t) \cdot \mathbf{P}(0)\rangle$ as a function of time. This calculation is performed using the model described in Section 4 and uses parameters that are derived from the femtosecond mid-infrared experiments shown in Figs. 2 and 4. The calculated $\langle\mathbf{P}(t) \cdot \mathbf{P}(0)\rangle$ consists of a dominant exponential component with a time constant of $7.85 \mathrm{ps}$, shown in (a), and a much weaker non-exponential component, highlighted in (b) by substracting $A \mathrm{e}^{-t / 7.85}$ ps from $\langle\mathbf{P}(t) \cdot \mathbf{P}(0)\rangle$. The values of $A$ and $B$ are 0.955 and 0.045 , respectively.

contribution to the dielectric relaxation, different Debye time constants ranging from less than $200 \mathrm{fs}$ up to a few picoseconds have been reported [10-16], which agrees quite well with the calculated result. The fact that the different studies do not agree on the precise value of the time constant may very well result from the small amplitude and non-exponential character of the fast component.

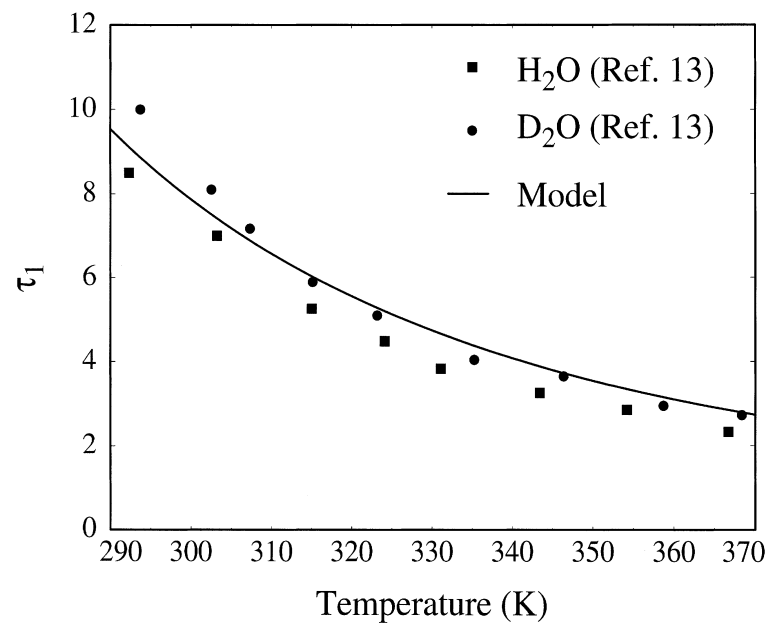

Fig. 8. Time constant of the slow contribution to the dielectric response of $\mathrm{H}_{2} \mathrm{O}$ and $\mathrm{D}_{2} \mathrm{O}$ as a function of temperature, measured with far-infrared spectroscopy (reproduced with the courtesy of the authors of Ref. [13]). Also shown is the temperature dependence of the calculated $\langle\mathbf{P}(t) \cdot \mathbf{P}(0)\rangle$ of HDO in $\mathrm{D}_{2} \mathrm{O}$, obtained using the model described in Section 4.

The reorientational motion of water molecules has also been studied with NMR techniques. The longitudinal relaxation time constant of the spin polarization can be related to the second-order correlation function $C_{2}(t)=\left\langle P_{2}(\mathbf{e}(t) \cdot \mathbf{e}(0))\right\rangle=$ $\mathrm{e}^{-t / \tau_{2}}$. In NMR studies, a value for $\tau_{2}$ of $2.6 \mathrm{ps}$ was found [35], which corresponds to a value for $\tau_{1}$ of $7.8 \mathrm{ps}$, in excellent agreement with the calculated value for $\tau_{1}$ of $7.85 \mathrm{ps}$.

In Fig. 8, the time constant of the exponential decay to which the calculated $\langle\mathbf{P}(t) \cdot \mathbf{P}(0)\rangle$ converges is plotted as a function of temperature and compared with the results of recent accurate farinfrared spectroscopic measurements of the slow component of the dielectric relaxation of water and deuterated water (reproduced with the courtesy of the authors of Ref. [13]). It is seen that the time constant of the dielectric relaxation decreases from $9.5 \mathrm{ps}$ at $290 \mathrm{~K}$ to $2.7 \mathrm{ps}$ at $370 \mathrm{~K}$. The calculated result for $\mathrm{HDO}$ in $\mathrm{D}_{2} \mathrm{O}$ is somewhat in between the measurements for $\mathrm{H}_{2} \mathrm{O}$ and $\mathrm{D}_{2} \mathrm{O}$ and quite accurately follows the temperature dependence of the experimental values.

In previous far-infrared $(\mathrm{THz})$ spectroscopic experiments, it was found that the temperature dependence of the dielectric relaxation cannot be 
modeled as an activated process [12]. At first sight, this may seem surprising since, according to Eq. (4.4), the reorientation rate is described as an activated process with an activation enthalpy that depends on the hydrogen-bond length. However, the overall dielectric relaxation time constant is not only determined by the relaxation rate at a particular hydrogen-bond length but also by the time constant of the stochastic modulation of the hydrogen-bond length and the fraction of molecules for which the reorientation is fast. This leads to a more complex temperature dependence for the overall dielectric relaxation rate. Fitting the observed temperature dependence of the dielectric relaxation as an activated process results in an activation enthalpy that decreases with temperature [12]. This can be understood as follows: At low temperatures, the fraction of molecules that show fast reorientation is very small but increases strongly with temperature. As a result, the observed overall reorientation rate increases rapidly with temperature, thereby suggesting a large activation enthalpy for the overall rate. However, at higher temperatures, the fraction of molecules showing fast reorientation is already substantial and increases negligibly upon further increasing the temperature. As a result, the overall reorientation rate increases much more slowly with temperature, which suggests a small activation enthalpy. Clearly, the definition of an activation enthalpy for the observed relaxation time is not very meaningful since such a definition neglects the strong dependence of the reorientation rate on the hydrogen-bond length and the stochastic modulation of this length. Only by including these effects, as is done in the model of Section 4, the observed complicated temperature dependence can be accounted for.

It was also found that the temperature dependence of the dielectric relaxation scales well with the viscosity of liquid water following the DebyeStokes-Einstein equation [12]. This strongly suggests that the viscosity of liquid water is inversely related to the fraction of molecules for which the hydrogen-bond interaction is weak and for which the reorientation is fast. Finally, it was found that the temperature dependence of the dielectric relaxation constant suggests a critical temperature
[36]. The temperature dependence of many properties of liquid water, including the dielectric relaxation, show a temperature dependence following $\left|T-T_{\mathrm{c}}\right|^{-\gamma}$ (Speedy-Angel plot) with a critical temperature $T_{\mathrm{c}}$ of $228 \mathrm{~K}$. The model of Section 4 does not contain a critical temperature. On the other hand, it is quite likely that at temperatures near $230 \mathrm{~K}$, the fraction of molecules for which the reorientation can be fast becomes negligible, not only because the temperature-dependent reorientation rate decreases but also because there will be a narrowing of the distribution of hydrogen-bond lengths. As a result, the effective reorientation time constant can indeed become infinitely large.

\section{Conclusions}

We studied the orientational dynamics of liquid water using femtosecond mid-infrared saturation spectroscopy on the $\mathrm{O}-\mathrm{H}$ stretching mode of HDO dissolved in $\mathrm{D}_{2} \mathrm{O}$. The advantage of this technique over conventional techniques (dielectric relaxation, stimulated Raman) is that the orientational dynamics of different subensembles of the HDO molecules can be selectively investigated by tuning the frequency of the excitation pulses through the inhomogeneously broadened absorption band of the $\mathrm{O}-\mathrm{H}$ stretch vibration. It is observed that the dynamics of the reorientation consists of a weak initial fast component followed by a much stronger slow component.

We found that the observed orientational dynamics can be well described with a model in which the reorientation rate depends on the length of the $\mathrm{O}-\mathrm{H} \cdots \mathrm{O}$ hydrogen bond and in which the stochastic modulation of the length of this bond is accounted for. This stochastic modulation has a characteristic time scale of $500 \mathrm{fs}$ and is described with a model in which the $\mathrm{O}-\mathrm{H}$... O hydrogen bond is described as an overdamped Brownian oscillator.

The model also provides a quantitative description of the dielectric response of liquid water, as is observed in dielectric relaxation and farinfrared spectroscopic studies. It also explains why the temperature dependence of the dielectric re- 
sponse cannot be described as a simple activated process. The good agreement between the model and the experimental observations enables a microscopic interpretation of the fast and the slow component that are observed in all orientational and dielectric relaxation studies on liquid water. After anisotropic excitation with an ultrashort laser pulse or after switching the static electric field in a dielectric relaxation experiment, the water molecules for which the $\mathrm{O}-\mathrm{H}$. . O hydrogen bond is long (weak) will show a direct, rapid reorientation. After approximately $1 \mathrm{ps}$, the rapid stochastic modulation of the length of the $\mathrm{O}-\mathrm{H} \cdots \mathrm{O}$ hydrogen-bond has led to an equilibration of the anisotropic distribution that is concentrated at short $\mathrm{O}-\mathrm{H} \cdots \mathrm{O}$ hydrogen-bond lengths for which the reorientation is slow. For these water molecules, the hydrogen-bond should first be lengthened (weakened) before reorientation can occur. This mechanism of reorientation has an associated effective reorientation time scale that decreases with increasing fractions of fast reorienting molecules and decreasing time constants for the reorientation and the stochastic modulation of the hydrogenbond length.

\section{Acknowledgements}

The research presented in this paper is a part of the research program of the Stichting Fundamenteel Onderzoek der Materie (Foundation for Fundamental Research on Matter) and was made possible by financial support from the Nederlandse Organisatie voor Wetenschappelijk Onderzoek (Netherlands Organization for the Advancement of Research).

\section{References}

[1] C.W. Robertson, D. Williams, J. Opt. Soc. Am. 61 (1971) 1316.

[2] J.B. Hasted, S.K. Husain, F.A.M. Frescura, J.R. Birch, Chem. Phys. Lett. 118 (1985) 622.

[3] G.E. Walrafen, J. Phys. Chem. 94 (1990) 2237.

[4] L. Thrane, R.H. Jacobsen, P.U. Jepsen, S.R. Keiding, Chem. Phys. Lett. 240 (1995) 330.
[5] H. Graener, G. Seifert, A. Laubereau, Phys. Rev. Lett. 66 (1991) 2092.

[6] S. Palese, S. Mukamel, R.J.D. Miller, W.T. Lotshaw, J. Phys. Chem. 100 (1996) 10380.

[7] W.B. Bosma, L.E. Fried, S. Mukamel, J. Chem. Phys. 98 (1993) 4413.

[8] M. Souaille, J.C. Smith, Mol. Phys. 87 (1996) 1333.

[9] M.N. Afsar, J.B. Hasted, J. Opt. Soc. Am. 67 (1977) 902.

[10] J. Barthel, K. Bachhuber, R. Buchner, H. Hetzenauer, Chem. Phys. Lett. 165 (1990) 369.

[11] J.T. Kindt, C.A. Schuttenmaer, J. Phys. Chem. 100 (1996) 10373.

[12] C. Rønne, L. Thrane, P.-O. Åstrand, A. Wallqvist, S. Keiding, J. Chem. Phys. 107 (1997) 5319.

[13] C. Rønne, P.O. Åstrand, S.R. Keiding, Phys. Rev. Lett. 82 (1999) 2888.

[14] S. Palese, L. Schilling, R.J.D. Miller, P.R. Staver, W.T. Lotshaw, J. Phys. Chem. 98 (1994) 6308.

[15] C.J. Montrose, J.A. Bucaro, J. Marshall-Coakley, T.A. Litovitz, J. Chem. Phys. 60 (1974) 5025.

[16] Y.J. Chang, E.W. Castner Jr., J. Chem. Phys. 99 (1993) 7289.

[17] S. Woutersen, U. Emmerichs, H.J. Bakker, Science 278 (1997) 658.

[18] S. Woutersen, U. Emmerichs, H.-K. Nienhuys, H.J. Bakker, Phys. Rev. Lett. 81 (1998) 1106.

[19] G.M. Gale, G. Gallot, F. Hache, N. Lascoux, S. Bratos, J.-C. Leicknam, Phys. Rev. Lett. 82 (1999) 1086.

[20] S. Woutersen, H.J. Bakker, Phys. Rev. Lett. 83 (1999) 2077.

[21] Y. Marechal, A. Witkowski, J. Chem. Phys. 48 (1968) 3697.

[22] A. Witkowski, J. Chem. Phys. 52 (1970) 4403.

[23] S. Bratos, J. Chem. Phys. 63 (1975) 3499.

[24] B. Boulil, O. Henri-Rousseau, Chem. Phys. 126 (1988) 263.

[25] S. Bratos, J.-C. Leicknam, J. Chem. Phys. 103 (1996) 4887.

[26] A.I. Burshtein, A.Y. Sivachenko, J. Chem. Phys. 112 (2000) 4699.

[27] A. Staib, J.T. Hynes, Chem. Phys. Lett. 204 (1993) 197.

[28] S. Mukamel, Principles of Nonlinear Optical Spectroscopy, Oxford University Press, New York, 1995.

[29] F. Franks (Ed.) Water, A Comprehensive Treatise Plenum, New York, 1972.

[30] A. Luzar, D. Chandler, Phys. Rev. Lett. 76 (1996) 928.

[31] A.I. Burshtein, V.S. Malinovsky, J. Opt. Soc. Am. B 8 (1991) 1098.

[32] G.E. Walrafen, M.R. Fisher, M.S. Hokmabadi, W.-H. Yang, J. Chem. Phys. 85 (1986) 6970.

[33] J. Teixeira, M.-C. Bellisent-Funel, S.H. Chem, A.J. Dianoux, Phys. Rev. A 31 (1985) 1931.

[34] S. Woutersen, Femtosecond vibrational dynamics in hydrogen-bonded systems, Thesis, University of Amsterdam, 1999.

[35] D.W.G. Smith, J.G. Powles, Mol. Phys. 10 (1966) 451.

[36] R.J. Speedy, C.A. Angell, J. Chem. Phys. 65 (1976) 851. 